\title{
25 Research Soure \\ Preparation of cell-sized water-in-oil droplets for in vitro reconstitution of biological processes in cellular compartments
}

\section{Makito Miyazaki ( $\square$ makito.miyazaki@aoni.waseda.jp )}

Department of Physics, Faculty of Science and Engineering, Waseda University, 3-4-1 Okubo, Shinjukuku, Tokyo 169-8555, Japan

\section{Masataka Chiba}

Department of Physics, Faculty of Science and Engineering, Waseda University, 3-4-1 Okubo, Shinjukuku, Tokyo 169-8555, Japan

\section{Shin'ichi Ishiwata}

Department of Physics, Faculty of Science and Engineering, Waseda University, 3-4-1 Okubo, Shinjukuku, Tokyo 169-8555, Japan; Waseda Bioscience Research Institute in Singapore (WABIOS), 11 Biopolis Way, \#05-01/02 Helios, Singapore 138667, Singapore

\section{Method Article}

Keywords: water-in-oil droplet, liposome, artificial cell, reconstitution, cytoskeleton, actin, myosin, contractile ring

Posted Date: March 24th, 2015

DOI: https://doi.org/10.1038/protex.2015.029

License: (c) (1) This work is licensed under a Creative Commons Attribution 4.0 International License. Read Full License 


\section{Abstract}

This protocol presents a method for encapsulation of purified proteins into cell-sized water-in-oil droplets surrounded by a phospholipid monolayer, which can be broadly applied to studies to reconstitute biological processes in cellular compartments.

\section{Introduction}

In the past decade,_in vitro_ reconstitution of biological processes from component molecules has been regarded as a powerful approach to uncover the underlying design principles of cells ${ }^{1}$. This bottom-up approach enables independent, precise control of biochemical parameters, such as protein composition and concentration, and physical parameters, such as boundary conditions and system size. Thus, the minimal necessary components and specific physical conditions essential for realizing biological functions can be identified. Here, we present a simple protocol for encapsulating purified proteins into cell-sized water-in-oil droplets surrounded by a phospholipid monolayer to mimic the cell boundary. This water-in-oil droplet system has been extensively utilized to investigate the effects of cellular microenvironments on various biological processes, including the self-organization of cytoskeletal architecture $^{2-8}$ and gene expression ${ }^{9,10}$. We adopted this system to elucidate the direct contribution of the cellular boundary to the assembly of the cytokinetic ring, and clarified that actin filaments and myosin motors can self-organize into ring-shaped contractile bundles when they are confined to a cell-sized spherical space, even without spatiotemporal regulatory signals ${ }^{11}$. The protocol described below was according to our previous reports ${ }^{11,12}$.

\section{Reagents}

- Phospholipids $\backslash($ Avanti Polar Lipids, Alabama, USA), e.g. L-a-phosphatidylcholine from chicken egg yolk \(egg PC; 840051P), 1,2-dioleoyl-sn-glycero-3-phosphocholine \(DOPC; 850375P), or 1,2-dioleoyl-snglycero-3-phosphoethanolamine \(DOPE; 850725P) • Chloroform \(034-02603; Wako Chemicals, Osaka Japan) • Molecular sieves $\backslash(4 A ;$ 04167-75; Nacalai Tesque, Kyoto, Japan) • Mineral oil $\backslash(23306-84$; Nacalai Tesque, Kyoto, Japan) $\cdot$ Valap $\backslash($ a mixture of Vaseline, lanolin, and paraffin at equal weight ratios) $\cdot$ Purified protein solution

\section{Equipment}

- Vacuum desiccator - Dry bath incubator - Vortex mixer - Water bath sonicator \(AS12GTU; As One, Osaka, Japan) • Glass test tube $\backslash(1.5 \mathrm{~mL}$ : 0407-03; Maruemu, Osaka, Japan) • Microcentrifuge tube $\backslash(1.5$ $\mathrm{mL}$ : 0030120086; Eppendorf, Hamburg, Germany) • Heat-resistant sealing film \(DuraSeal; Diversified Biotech, Massachusetts, USA $) \cdot$ Silicone-coated coverslip $\backslash\left(24 \times 36 \mathrm{~mm}^{2}\right.$, thickness No. 1: custom-order; Matsunami Glass, Tokyo, Japan; or equivalent hydrophobic-surfaced coverslip) • Normal coverslip \(18 $\times$ $18 \mathrm{~mm}^{2}$, thickness No. 1: C218181; Matsunami Glass, Tokyo, Japan) • Double-sided adhesive tape \ (thickness: $\sim 100 \mu \mathrm{m}) \cdot$ Floating microcentrifuge tube rack • Micropipettes $•$ Optical microscope 


\section{Procedure}

**PREPARATION OF A LIPID DRY FILM** 1. Dissolve phospholipids in chloroform \(dehydrated with molecular sieves) to a final concentration of $20 \mathrm{mM}$. 2. Dispense the lipid solution into glass test tubes $\backslash$ (50 $\mu \mathrm{L}$ per tube). 3. Place the test tubes in a vacuum desiccator. 4. Evacuate overnight at room temperature in the dark to completely remove chloroform $\backslash$ (Fig. 1a). 5. Store lipid dry films in a vacuum desiccator at room temperature in the dark. The lipid dry films should be used within 1 month. Tips: Phospholipids should be stored at $-20^{\circ} \mathrm{C}$ under a nitrogen atmosphere in the dark. ${ }^{*}$ PREPARATION OF LIPID-OIL MIXTURE** 6. Mix mineral oil by gentle inversion just before use. 7. Collect a lipid dry film sample from a vacuum desiccator. 8. Place $1 \mathrm{~mL}$ of mineral oil into the glass test tube. 9. Seal the top of the tube with a heat-resistant sealing film $\backslash$ (Fig. 1b). 10. Incubate the tube at $80^{\circ} \mathrm{C}$ for $\sim 15 \mathrm{~min}$ in a dry bath incubator. 11. Dissolve the lipid dry film by vortexing $\backslash(\sim 10 \mathrm{~s}$; Fig. 1c). 12. If the lipid dry film remains at the bottom of the tube, repeat steps 10 and 11.13. Set the tube in a floating rack and float in a water bath sonicator. 14 . Sonicate the tube for $90 \mathrm{~min}$ at $60^{\circ} \mathrm{C}$ and $60 \mathrm{~W}$ power $\backslash$ (Fig. $1 \mathrm{~d}$ ). 15. Immediately after sonication, mix the lipid-oil mixture by vortexing $\backslash(\sim 10 \mathrm{~s})$. 16. Cool to room temperature and incubate overnight at room temperature in the dark. 17. The concentration of lipids in oil is $1 \mathrm{mM}$. Store the lipid-oil mixture at room temperature in the dark, and use within 1 week of preparation. **PREPARATION OF A FLOW CHAMBER ** 18. Place two pieces of double-sided adhesive tape $\backslash$ (thickness: $100 \mu \mathrm{m}$ ) onto a siliconecoated coverslip with a $8 \mathrm{~mm}$ gap between them. 19. Place a normal coverslip on top of the gap, to produce a chamber of $18 \mathrm{~mm} \times 8 \mathrm{~mm} \times 100 \mu \mathrm{m} \backslash$ (see Fig. 2c). **ENCAPSLATION OF PROTEINS INTO WATER-INOIL DROPLETS** 20. Mix lipid-oil mixture by vortexing immediately before use. 21 . Transfer $80 \mu \mathrm{L}$ of the lipid-oil mixture into a $1.5-\mathrm{mL}$ microcentrifuge tube pre-equilibrated to a desired temperature. 22. Pipet 2 $\mu \mathrm{L}$ of protein solution directly into the lipid-oil mixture in the microcentrifuge tube $\backslash$ (Fig. 2a). 23. Mix the lipid-oil mixture and protein solutions by vortexing for 10-30 s to form water-in-oil droplets $\backslash$ (Fig. 2b). The droplet size can be controlled by optimizing the power and duration of vortexing. 24. Perfuse the water-inoil emulsion into a flow chamber \(Fig. 2c). 25. Seal the flow chamber with Valap \(Fig. 2d). 26. Observe the water-in-oil droplets using an optical microscope $\backslash$ (Fig. 3).

\section{Timing}

- Preparation of lipid dry films requires an overnight step. The lipid dry films should be stored in a vacuum desiccator at room temperature in the dark, and used within 1 month of preparation. Preparation of lipidoil mixture is performed in 1 day. The lipid-oil mixture should be stored at room temperature in the dark, and used within 1 week of preparation. Encapsulation of proteins in water-in-oil droplets takes $\sim 5$ min.

\section{References}

1. Liu, A. P. \& Fletcher, D. A. Biology under construction: in vitro reconstitution of cellular function._Nat. Rev. Mol. Cell Biol._ ${ }^{\star *} 10^{* \star}, 644-650 \backslash(2009)$. 2. Hase, M. \& Yoshikawa, K. Structural transition of actin filament in a cell-sized water droplet with a phospholipid membrane. _J. Chem. Phys._ **124**, $104903 \backslash$ (2006). 3. Claessens, M. M. A. E., Tharmann, R., Kroy, K. \& Bausch, A. R. Microstructure and viscoelasticity 
of confined semiflexible polymer networks. _Nat. Phys._ ${ }^{\star \star} 2^{\star \star}, 186-189 \backslash(2006)$. 4. Pinot, M. _et al._ Effects of confinement on the self-organization of microtubules and motors. _Curr. Biol._**19**, 954$960 \backslash(2009)$. 5. Negishi, M., Sakaue, T., Takiguchi, K. \& Yoshikawa, K. Cooperation between giant DNA molecules and actin filaments in a microsphere. _Phys. Rev. E_ $* \star 81 * \star, 051921 \backslash(2010)$. 6. Pinot, M. _et al._ Confinement induces actin flow in a meiotic cytoplasm. _Proc. Natl Acad. Sci. USA_**109**, 11705$11710 \backslash(2012)$. 7. Hazel, J._et al._ Changes in cytoplasmic volume are sufficient to drive spindle scaling. _Science_**342**, 853-856 \(2013). 8. Good, M. C., Vahey, M. D., Skandarajah, A., Fletcher, D. A. \& Heald, R. Cytoplasmic volume modulates spindle size during embryogenesis. _Science_**342**, 856-860 \} (2013). 9. Kato, A., Yanagisawa, M., Sato, Y. T., Fujiwara, K. \& Yoshikawa, K. Cell-sized confinement in microspheres accelerates the reaction of gene expression._Sci. Rep._ ${ }^{\star \star} 2^{\star \star}, 283 \backslash(2012)$. 10. Matsuura, T. _et al_. Effects of compartment size on the kinetics of intracompartmental multimeric protein synthesis. _ACS Synth. Biol._ ${ }^{\star \star} 1 * \star, 431-437 \backslash(2012)$. 11. Miyazaki, M., Chiba, M., Eguchi, H., Ohki, T. \& Ishiwata, S. Cell-sized spherical confinement induces the spontaneous formation of contractile actomyosin rings _in vitro_._Nat. Cell Biol._, in press. 12. Chiba, M., Miyazaki, M. \& Ishiwata, S. Quantitative analysis of the lamellarity of giant liposomes prepared by the inverted emulsion method. _Biophys. J._ ${ }^{\star *} 107 * \star, 346-354$ $\backslash(2014)$.

\section{Acknowledgements}

This work was supported in part by a Sasakawa Scientific Research Grant from The Japan Science Society to M.M., Grants-in-Aid for Young Scientists $\backslash(B)$ and Scientific Research on Innovative Areas to M.M., and Grants-in-Aid for Specially Promoted Research and Scientific Research \(S) to S.I. from the Ministry of Education, Culture, Sports, Science, and Technology of Japan.

\section{Figures}
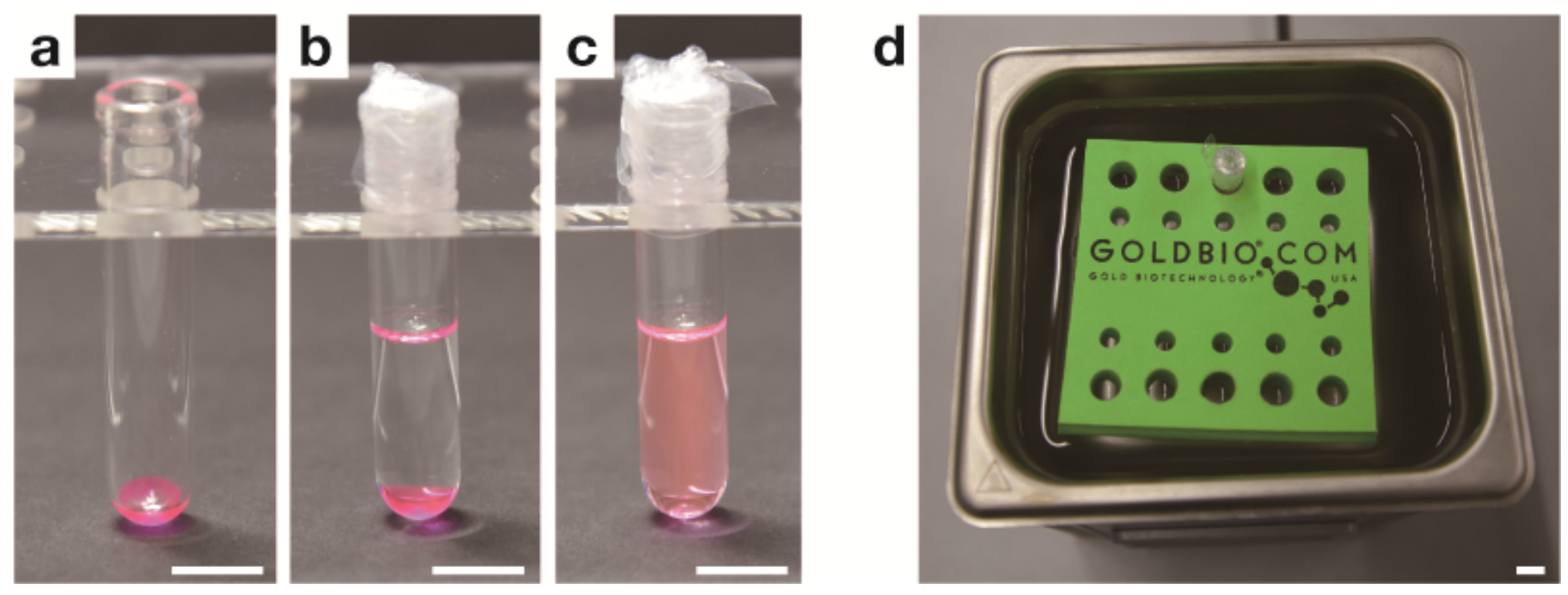

Figure 1 
Preparation of lipid-oil mixture. $<$ a

href="http://www.nature.com/protocolexchange/system/uploads/3571/original/fig1_v1_00.tif?

$1426826338 "><$ img

src="http://www.nature.com/protocolexchange/system/uploads/3571/original/fig1_v1_00.tif?

1426826338 " > </a> To demonstrate the process of dissolving a lipid dry film, egg PC containing $0.5 \%$

(mol/mol) of rhodamine-labelled phospholipids (1,2-dioleoyl-sn-glycero-3-phosphoethanolamine-N-

lissamine rhodamine B sulfonyl ammonium salt; 810150C, Avanti Polar Lipids, Alabama, USA) was used.

$\left({ }^{*}{ }^{*}\right)$ Formation of a lipid dry film at the bottom of a glass test tube by overnight evacuation. (*b*)

Overlaying a lipid dry film with mineral oil and sealing the tube top with a heat-resistant film. $\left({ }^{*} \mathrm{C}^{\star}\right)$

Dissolving the lipid dry film by heating and vortexing. $\left({ }^{*} \mathrm{~d}^{*}\right)$ Sonication in a water bath sonicator. Scale bars, $1 \mathrm{~cm}$.
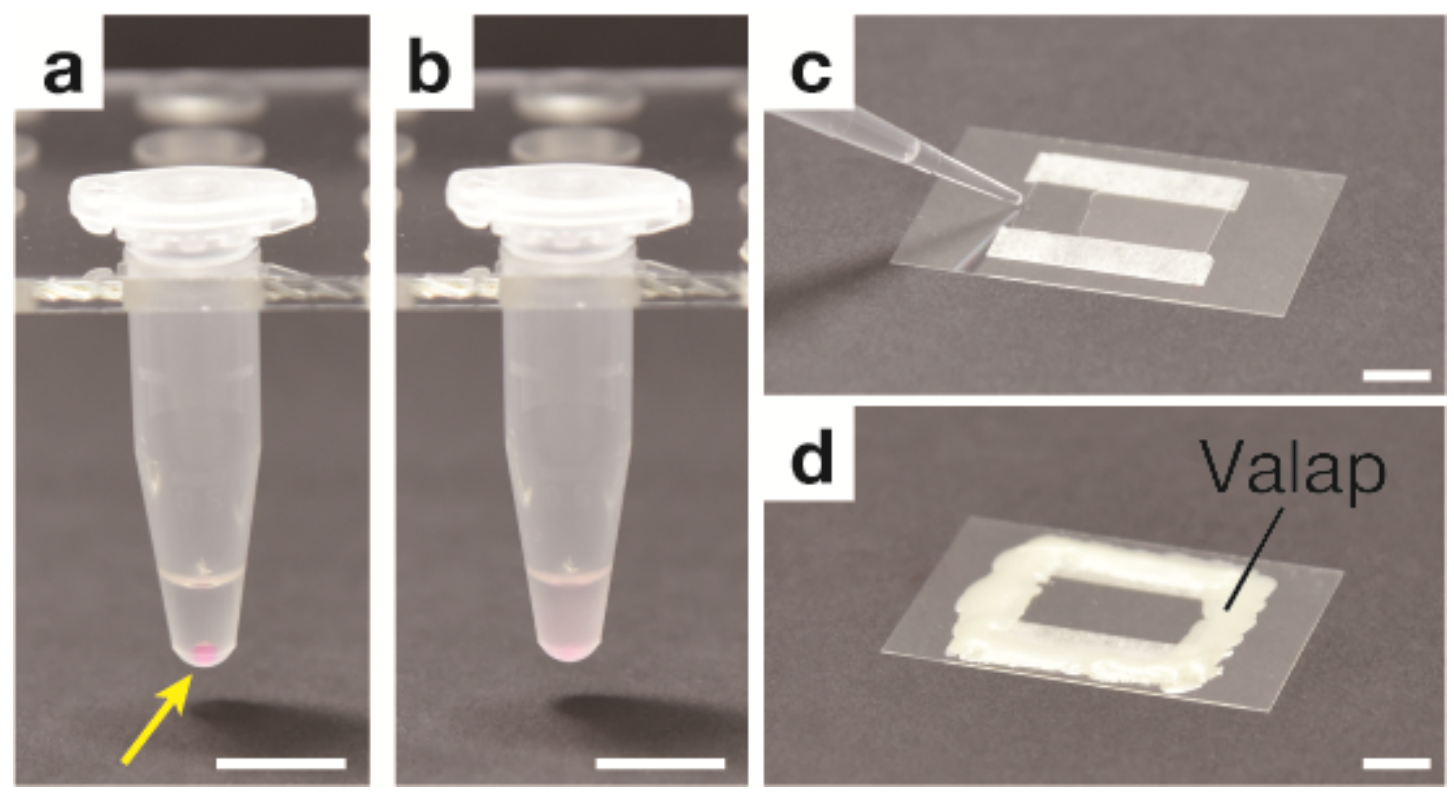

\section{Figure 2}

Encapsulation of proteins into cell-sized water-in-oil droplets. <a href="http://www.nature.com/protocolexchange/system/uploads/3573/original/fig2_v1_01.tif? $1426826871 "><$ img src="http://www.nature.com/protocolexchange/system/uploads/3573/original/fig2_v1_01.tif? $1426826871^{\prime \prime}></$ a > Tetramethylrhodamine-labelled bovine serum albumin (TMR-BSA, $5 \mathrm{mg} \mathrm{mL}^{\wedge}-1^{\wedge}$ ) in PBS was encapsulated. Egg PC was used for phospholipids. (*a*) Before vortexing. The yellow arrow indicates the TMR-BSA solution in the lipid-oil mixture. (* $\left.{ }^{\star}\right)$ After vortexing. Many cell-sized water-in-oil droplets were formed. $\left({ }^{*} c^{*}\right)$ Perfusion of water-in-oil droplets into a flow chamber. $\left({ }^{*}{ }^{*}\right)$ Sealing the four sides of the flow chamber with Valap to prevent flow and evaporation of water from the droplets. Scale bars, $1 \mathrm{~cm}$. 


\section{Bright-field}

TMR-BSA

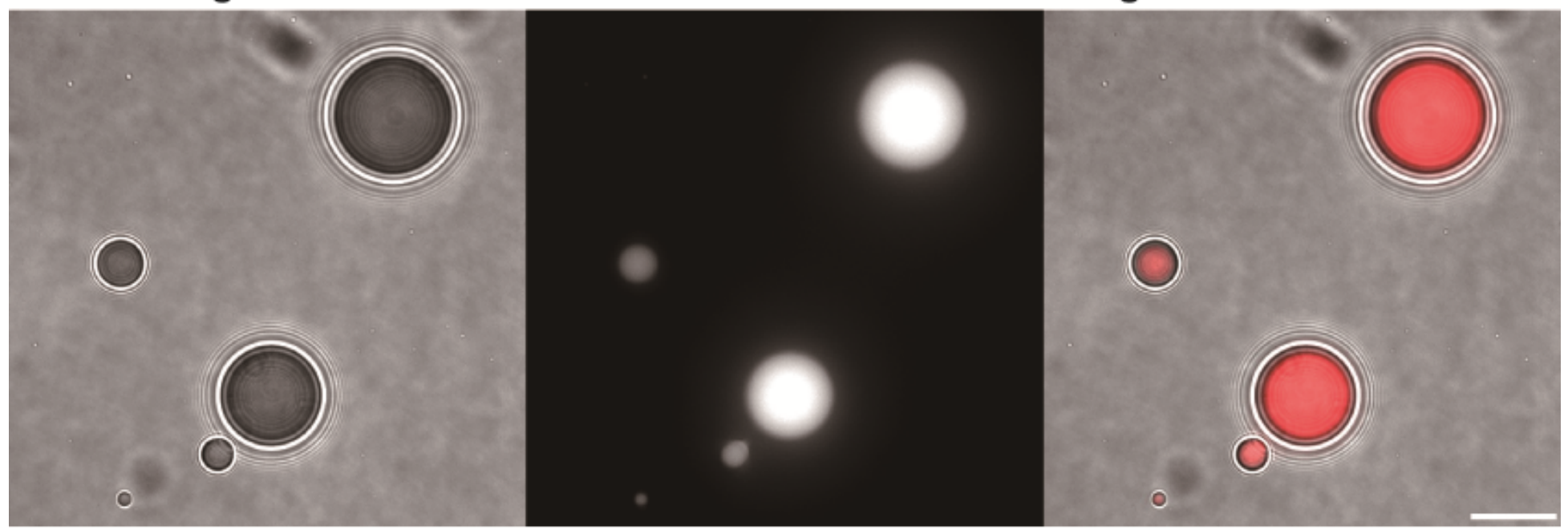

\section{Figure 3}

Observation of water-in-oil droplets under an optical microscope. <a href="http://www.nature.com/protocolexchange/system/uploads/3577/original/fig3_v1_01.tif? $1426831968 "><$ img src="http://www.nature.com/protocolexchange/system/uploads/3577/original/fig3_v1_01.tif? 1426831968 " $></ a>$ A bright-field image, a fluorescence image, and the merged image of droplets containing $5 \mathrm{mg} \mathrm{mL} \mathrm{mL}^{\wedge} 1^{\wedge}$ TMR-BSA in PBS. An epifluorescence inverted microscope (custom-built) equipped with a $\times 60$ objective (PlanApo $\times 60$ NA 1.45 Oil TIRFM, Olympus) and an electron-multiplying CCD (charge-coupled device) camera (iXon 3, Andor Technology) was used. Phospholipid, egg PC; scale bar, $20 \mu \mathrm{m}$. 